\title{
The Effect of Nanoparticle Surface State on Trap Level Distribution of Polyimide/Aluminum Nitride-montmorillonite Nanocomposite Films
}

\author{
Yuanyuan $\quad$ LIU $^{1}$, Jinghua $\quad$ YIN $^{1 *}$, Tianyi $\quad \mathrm{JI}^{2}$, Duo $\quad$ SUN $^{1}$, Xiaoxu $\quad$ LIU $^{2}$ \\ ${ }^{1}$ Key Laboratory of Engineering Dielectrics and Its Application Ministry of Education, Harbin University of Science and \\ Technology, Harbin, PR China \\ ${ }^{2}$ Institute of Advanced Composite Material Technology, Heilongjiang University of Science and Technology, Harbin, PR \\ China \\ crossref http://dx.doi.org/10.5755/j01.ms.25.1.19144
}

Received 27 September 2017; accepted 19 January 2018

\begin{abstract}
The electrical properties of polyimide (PI) nanocomposites, which are widely used in microelectronic industry and electrical engineering fields, strongly depend on the surface state of nanoparticles. To explore this dependence, the aluminum nitride (AIN) nanoparticles were treated by $\gamma$-aminopropyltriethoxysilane coupling agent, while PI/MMT, PI/AlN, and PI/AIN-MMT nanocomposite films doped by $5 \mathrm{wt} . \%$ of treated and untreated AlN nanoparticles were prepared by the in-situ polymerization process. The SEM and TEM results indicate that the untreated AIN nanoparticles are prone to accumulation in the polymer matrix, while those treated by the coupling agent are readily combined with the polyimide matrix, and their compatibility and dispersion exhibit a significant improvement. The trap level distributions of nanocomposite films were studied by the isothermal discharge current (IDC) method based on the charge decay theory linking IDC with the trap level density (TLD). The TLD and number of trapped charges of PI/AlN and PI/AIN-MMT films doped by treated AIN nanoparticles are found to be much higher than those of untreated ones. The TLD of the PI/AlN (treated) film is $6.490 \times 10^{23} \mathrm{eV} \cdot \mathrm{m}^{-3}$, which is about 2.27 times higher than that of pure PI film in the range of $0.9 \sim 1.1 \mathrm{eV}$, while the maximum TLD=9.370 $\times 10^{23} \mathrm{eV} \cdot \mathrm{m}^{-3}$ is observed in the PI/AIN (treated)-MMT film. Keywords: polyimide; AlN and MMT particles; trap level distribution; nanocomposite film.
\end{abstract}

\section{INTRODUCTION}

Polyimides are incredibly strong synthetic polymers that are also astoundingly heat and chemical resistant. Nanocomposites based on these polymers are widely applied in many different fields, such as insulation materials, frequency conversion motors, electronic devices and so on [1-3]. Polyimides contain a special imide ring consisting of dicarboxylic acid and diamine, which implies their superior mechanical properties, high-temperature, radiation and solvent resistance, etc. For the excellent performance of two-dimensional material such as montmorillonite (MMT), BN and graphene, [4-6] many studies on polyimide-doped nanoparticles have been carried out to improve its electrical properties, where application of montmorillonite (MMT) turned out to be quite lucrative. MMT is a very soft phyllosilicate group of minerals that form when they precipitate from water solution as microscopic crystals, known as clay. Thus, MMT with high horizontal-vertical $(\mathrm{H} / \mathrm{V})$ size ratio is a common lamellar silicate. When MMT is doped into a polymer, their interface consists of numerous organic or inorganic contact zones. Due to interaction between MMT edges, some MMT layers get together, and plate-like substances, which are relatively larger than spherical AlN nano-fillers, are squeezed out from the bulk material. It gives evidence to the model-based fact that nano-fillers are covered by interfacial regions that consist of the first and second layers according to the multi-core model [7].

\footnotetext{
${ }^{*}$ Corresponding author. Tel.: +86-451-86390777.
}

E-mail address: yinjinghua1 @126.com (J. Yin)
The partial discharge and volume resistance of polyimide nanocomposite film can be reduced by the synergistic effect of lamellar MMT and spherical AlN nanoparticles, resulting in the corona of nanocomposite film to be improved. Therefore, the research of the trap level depth and density is topical for the charge transmission in polymer [8].

Doping nanoparticles can obviously change the characteristics of polymer due to the interface effect between inorganic nanoparticles and polymer [9]. The effect of nanoparticle surface state on the electrical properties attracted much attention of researchers. The surfaces of inorganic nanoparticles can be treated by coupling agent to change the compatibility and dispersion of nanoparticles in the polymer matrix. The inorganic nanoparticles can be closely combined with the polyimide matrix, inhibiting the auto-polymerization and enhancing the dispersion of inorganic nanoparticles.

In this study, five kinds of nanocomposite films doped by content of $5 \mathrm{wt} . \%$ were prepared by the in-situ polymerization process: PI/MMT, PI/AIN (treated), PI/AlN (untreated), PI/AlN (treated)-MMT and PI/AIN (untreated)-MMT. On the basis of the charge decay theory, a relationship between IDC density and TLD of nanocomposite films was assessed and the respective equation linking the trap level and density was proposed. The effect of nanoparticles surface state on traps of nanocomposite films was studied by the conventional IDC method introduced by Simmons to obtain the trap level distribution in the range of $10^{21} \sim 10^{23} \mathrm{eV} \mathrm{cm}^{-3}[10-12]$. The IDC and TLD of the nanocomposite films under study 
were assessed, and the interface trap effect relative to the surface state of AlN nanoparticles was analysed.

\section{EXPERIMENTAL}

The AlN nanoparticles with an average diameter of $<80 \mathrm{~nm}$ and purity of $99.9 \%$ (Beijing Chen Technology Co., Ltd) were used as the filler in the polyimide matrix. The N, N-dimethylacetamide (DMAC) was used as a solvent, while the 4,4'-oxydianiline (ODA) and pyromellitic dianhydride (PMDA) purchased from either Sinopharm Chemical Reagent Co., Ltd or Shanghai Chemical Reagent Co., Ltd were used to form the polyamic acid. The $\gamma$-aminopropyltriethoxysilane $\mathrm{KH}-550$ (Shanghai Chemical Reagent Co., Ltd) was used as a coupling agent to treat AlN nanoparticles. The layered montmorillonite (MMT) with an average lamination thickness of $25 \mathrm{~nm}$ and length of $2 \sim 5 \mu \mathrm{m}$ (Shanghai Chemical Reagent Co., Ltd) was used as an intercalator.

\subsection{Treating AIN nanoparticles with KH-550}

The surfaces of AlN nanoparticles were treated with $\mathrm{KH}-550$, according the following procedure:

1. Placing $5.0 \mathrm{~g}$ of AlN nanoparticles into a drying oven at $100{ }^{\circ} \mathrm{C}$ for $30 \mathrm{~min}$;

2. Adding coupling agent of $0.416 \mathrm{~g}$ and $10 \mathrm{ml}$ anhydrous ethanol to a three-opening flask;

3. Placing the AlN nanoparticles into the above mixed solution and injecting $40 \mathrm{ml}$ anhydrous ethanol into the three-opening flask to condense in reflux;

4. Mixing $3 \mathrm{~h}$ until reacting completely in a water bath at $80^{\circ}$;

5. Cooling to room temperature and then placing into a drying oven at $100{ }^{\circ} \mathrm{C}$ for $8 \mathrm{~h}$;

6. Grinding evenly in a mill.

\subsection{Film preparation}

On the basis of in-situ polymerization process, five kinds of films doped by AlN nanoparticles of $5 \mathrm{wt} \%$ (PI/MMT, PI/AIN (treated), PI/AIN (untreated), PI/AIN (treated)-MMT and PI/AIN (untreated)-MMT), and pure PI film were prepared. The synthesis process is schematically described in Fig. 1.

1. The treated AIN nanoparticles of $0.165 \mathrm{~g}$ and DMAC of $40 \mathrm{ml}$ are placed into a three-opening flask and then stirred in an ultra-sonic bath. Similarly, another flask with added untreated AlN nanoparticles and DMAC is also stirred, with application of mechanical stir and ultrasonic waves to obtain stable suspensions.

2. The ODA of $3.6227 \mathrm{~g}$ is placed into the flasks to completely dissolve in the suspensions by mechanical stir and ultrasonic waves.

3. The PMDA of $4.0673 \mathrm{~g}$ is subdivided into five portions, each being consequently added into the suspension until completely dissolving and obviously climbing stirring pole under rapid stirring to form polyamic acid (PAA). Thereafter, the obtained PAA is added to the suspension and stirred for $4 \mathrm{~h}$ to obtain a yellow mixture solution.

4. The solution is scraped on a glass plate by a metal mold and then heated to $80{ }^{\circ} \mathrm{C}$ in air for $12 \mathrm{~h}$, in order to get a rigid film.

5. The film is heated with gradually increased temperature to remove DMAC and promote imidization from PAA to PI. According to the heating steps and holding times listed in Table 1, when the temperature is $280{ }^{\circ} \mathrm{C}$, the imidization starts to happen, when reaching $350{ }^{\circ} \mathrm{C}$, the imidization is more complete. The resulting films with thickness of about $100 \mu \mathrm{m}$ are cooled to room temperature.

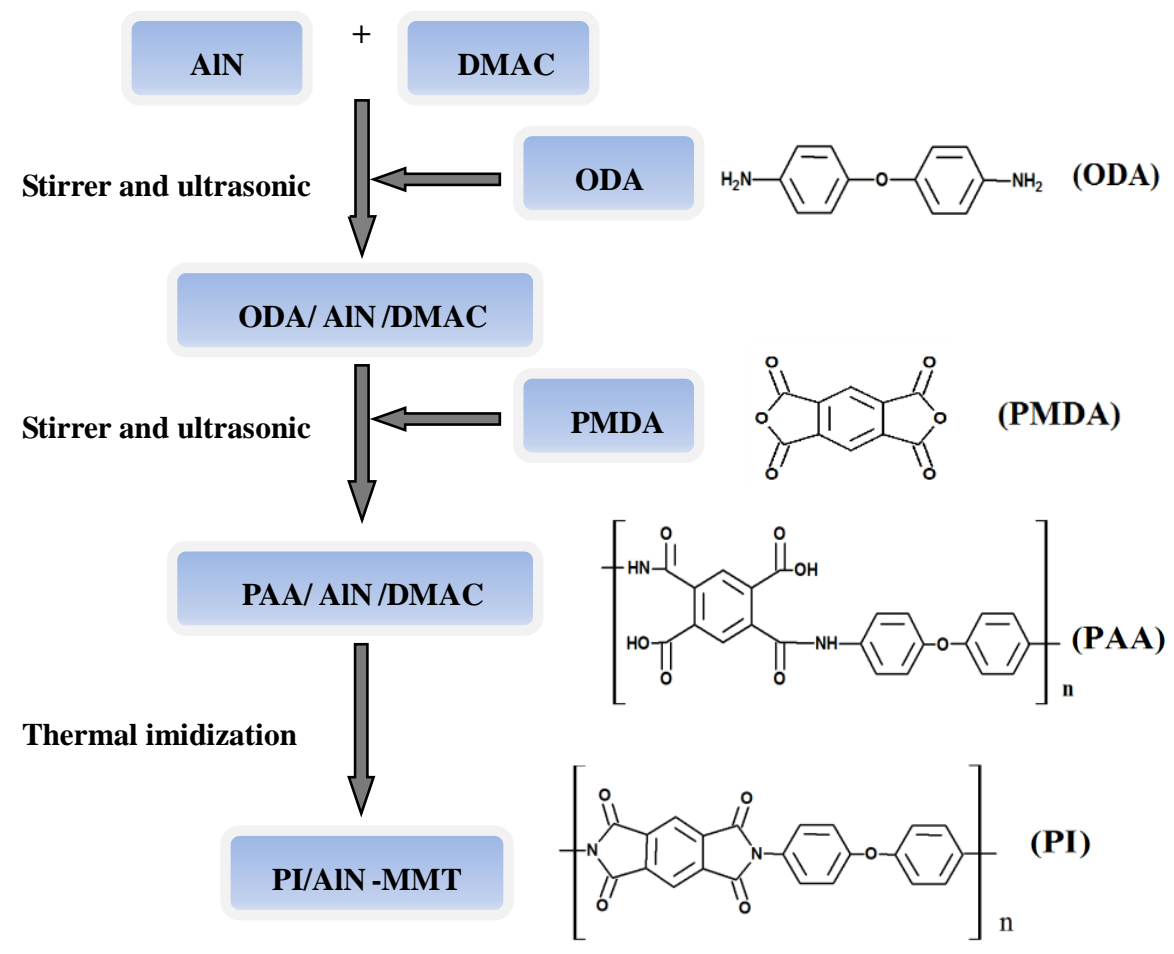

Fig. 1. The preparation process of the nanocomposite films 
Table 1. The heating steps to remove DMAC and promote imidization from PAA to PI

\begin{tabular}{|l|c|c|c|c|c|c|c|}
\hline Heating steps & 1 & 2 & 3 & 4 & 5 & 6 & 7 \\
\hline Temperature, ${ }^{\circ} \mathrm{C}$ & 120 & 150 & 180 & 210 & 270 & 310 & 350 \\
\hline Time, $\mathrm{h}$ & 0.5 & 0.5 & 0.5 & 0.5 & 0.5 & 0.5 & 1 \\
\hline
\end{tabular}

\subsection{AFM, SEM and TEM characterization}

In order to observe the aggregate state of AlN nanoparticles in the PI matrix, the surface roughness and the cross-sectional morphologies of the PI/AlN (untreated)-MMT and PI/AIN (treated)-MMT films were examined via a Nano Scope IIIA atomic force microscopy (AFM) and a FEI/Philips Quanta 200 scanning electron microscope (SEM) at $20 \mathrm{kV}$. The microstructure of treated AlN nanoparticles in the PI/AIN (treated)-MMT nanocomposite film was tested by a FEI Tecnai Spirit transmission electron microscope (TEM) at $120 \mathrm{kV}$.

\subsection{Isothermal discharge current test}

Rinsing the PI and nanocomposite films with acetone solution, the double sides of the nanocomposite films were evaporated with aluminum electrodes of $4 \mathrm{~cm}$ diameter. The films were shorted for $12 \mathrm{~h}$ in a DHJ-9140A electric heating constant-temperature drying box at $150{ }^{\circ} \mathrm{C}$. Next, the treated samples were taken out and placed in an oven with a constant temperature of $50{ }^{\circ} \mathrm{C}$. The samples were polarized for $2 \mathrm{~h}$ by a constant DC electric field of $40 \mathrm{kV} / \mathrm{mm}$. Thereafter the samples were immediately shorted. The IDC characteristic curves of the nanocomposite films with thickness of $100 \mu \mathrm{m}$ were constructed by the IDC test system consisting of a DW-102-20F high voltage DC source, EST122 picoammeter and electrode test box (Fig. 2).

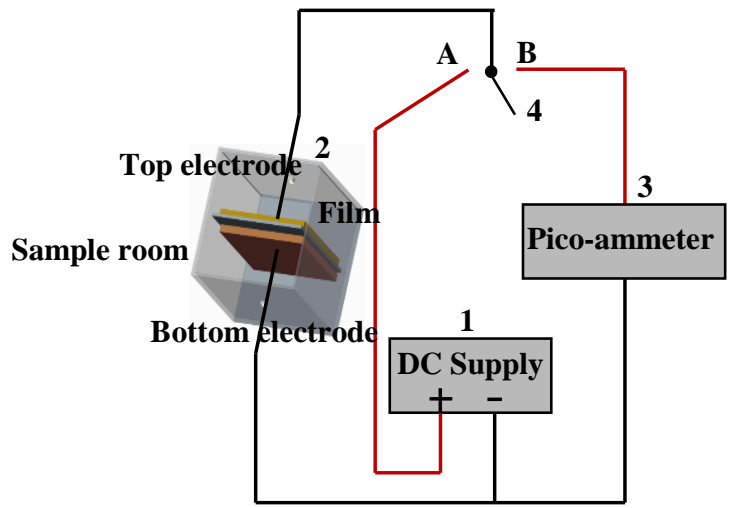

Fig. 2. Isothermal decay current test system: 1-DC source; 2 - pico-ammeter; 3 -test box; 4 -switch

\subsection{Theoretical analyses}

The sample is shorted when the external electric field is removed, the carriers in the low traps are released firstly, and then the carriers in the deep traps are released again. At room temperature, the short circuit current caused by the carriers released with decay time represents the trap level distribution of the sample surface. Assuming that the carriers released cannot be trapped again, the relation linking the trap level $E_{t}$, current density $J$ and trap density $N\left(E_{t}\right)$ can be expressed as in [13]:

$$
E_{t}=k T \ln (v t)
$$

$$
J(t)=\frac{q L k T}{2 t} f_{0}\left(E_{t}\right) N\left(E_{t}\right),
$$

where $k$ is the Boltzmann constant, $L$ is film thickness, $q$ is electronic charge, $T$ is the absolute test temperature. $N\left(E_{t}\right)$ is the distribution function of TLD and $f_{0}\left(E_{t}\right)$ is the probability of energy level $E_{m}$ being originally occupied by electrons, which is constant when the traps are full of electrons. The escaping frequency $v$ of the injected electrons is $10^{12} \mathrm{~s}^{-1}$ [14].

According to Eq. 2 and assuming that $f_{0}\left(E_{t}\right)=1 / 2$,

TLD is derived as

$$
N\left(E_{t}\right)=\frac{J(t) 4 t}{q L k T}
$$

The obtained Eq. 3 controls the relationship between TLD and trap level.

\section{RESULTS AND DISCUSSION}

Based on the charge decay theory, the trap level distributions of PI, PI/MMT, PI/AIN and PI/AlN-MMT films doped by treated and untreated AIN nanoparticles are studied by the IDC method. The IDC and interface trap effects relative to the surface state of AIN nanoparticles are also discussed.

\subsection{AFM, SEM and TEM images}

The cross-sectional morphologies and surface roughness of PI/AlN (untreated)-MMT and PI/AIN (treated)-MMT films doped by $5 \mathrm{wt} \%$ AlN are shown in Fig. 3. Here Fig. 3 a indicates that the AlN nanoparticles cluster together, which means that the untreated AlN nanoparticles cannot form a sufficient number of combinations with polyimide and MMT, resulting in an obvious surface roughness. As shown in Fig. 3 b, the compatibility of treated AlN nanoparticles, PI and MMT is improved, the dispersion of nanoparticles in the PI matrix is enhanced and the surface roughness is decreased. The AFM and SEM test results show that the surface state of treated inorganic nanoparticles promotes their combining with the PI matrix and can improve the compatibility between nanoparticles and PI matrix. The surface state can be modified by the coupling agent, since one end of molecular groups reacts with inorganic nanoparticles to form chemical bond and the other one reacts with PI matrix or forms physical windings, so that the AlN nanoparticles can readily combine with the PI matrix. Thus, the coupling agent can inhibit the auto-polymerization of inorganic nanoparticles and improve the dispersion of AlN nanoparticles. For closer examination of the aggregate state of AIN nanoparticles, 
the microstructure of treated AIN nanoparticles in the bulk of the PI/AIN (treated)-MMT nanocomposite film was subjected to TEM analysis, as is shown in Fig. $3 \mathrm{c}$. It can be seen that the treated AlN nanoparticles are uniformly dispersed in the PI matrix, where a better compatibility of PI and large-scale layered MMT or small-scale treated AlN nanoparticles is also revealed by the SEM image of the PI/AIN (treated)-MMT film.

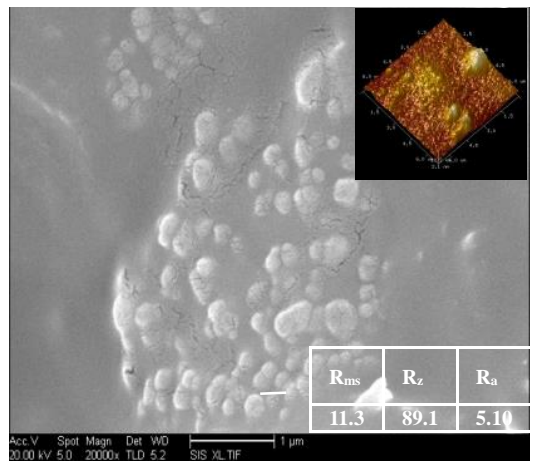

a
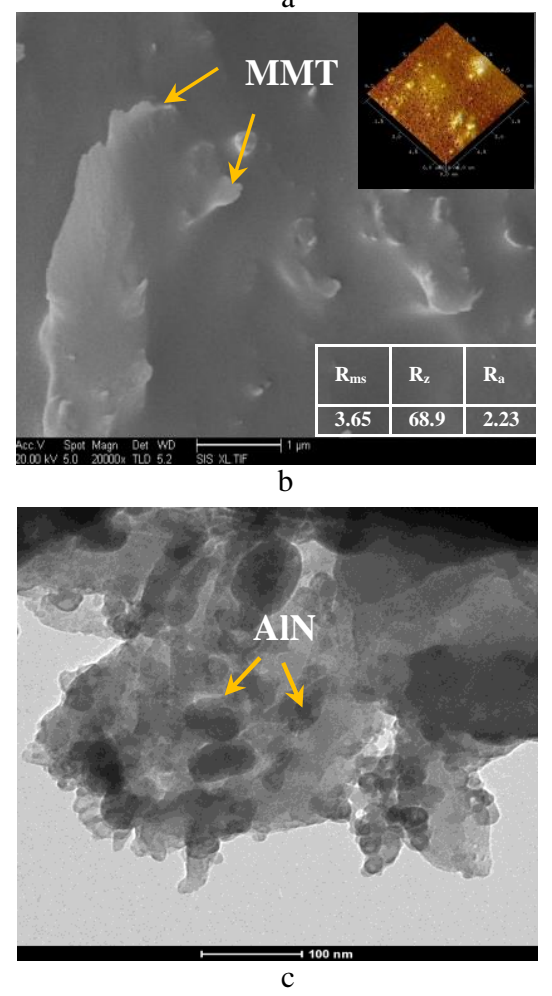

Fig. 3. The cross-sectional morphologies and AFMs of PI/AIN-MMT nanocomposite films doped by: a-5 wt.\% untreated AlN; b-treated AlN; c-TEM image of the $\mathrm{PI} / \mathrm{AlN}$ (treated)-MMT nanocomposite film

\subsection{IDC curves and trap level distribution}

The characteristic curves of the isothermal decay current vs. time (I-t) for PI, PI/MMT, PI/AlN (treated), PI/AlN (untreated), PI/AlN (treated)-MMT and PI/AlN (untreated)-MMT films and their trap level distributions were constructed for the test with a DC electric field of 40 $\mathrm{kV} / \mathrm{mm}$ exerted on the samples (Fig. 4). As shown in Fig. 4 a, the IDCs of all nanocomposite films are higher than that of PI until the decay current reaches stability, where the IDC of pure PI film does not vary with time anymore. In addition, the IDC of PI/AIN (treated)-MMT film exceeds not only that of the untreated one but also those of PI/MMT, PI/AIN (treated) and PI/AIN (untreated) films. The IDC of PI/AIN (treated) is higher than that of the PI/AIN (untreated) but is lower than those of PI/MMT and PI/AlN (untreated)-MMT films. Fig. $4 \mathrm{~b}$ shows that the trap level densities of the five nanocomposite films under study are significantly improved, as compared to the PI film, where the maximum TLD $=6.490 \times 10^{23} \mathrm{eV} \mathrm{m}^{-3}$ of the PI/AlN (treated) is about 2.27 times higher than that of pure PI film as shown in Table 2.

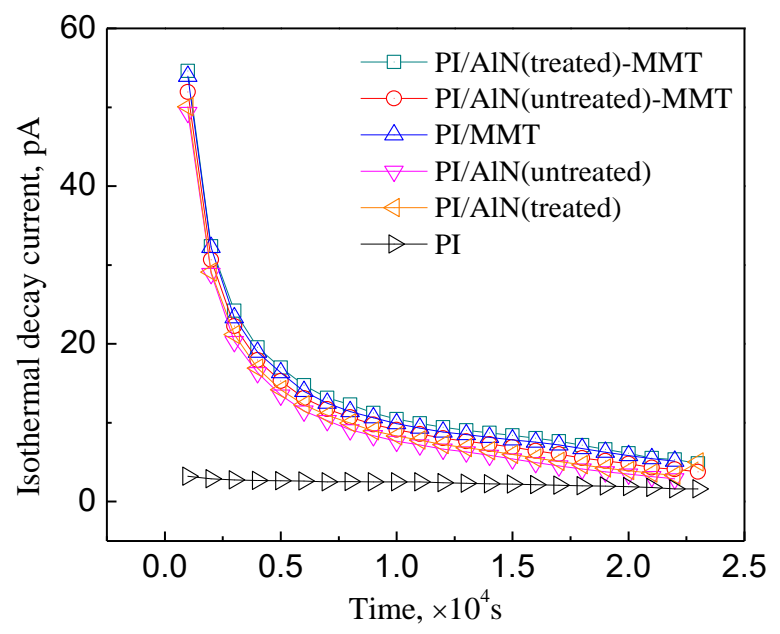

a

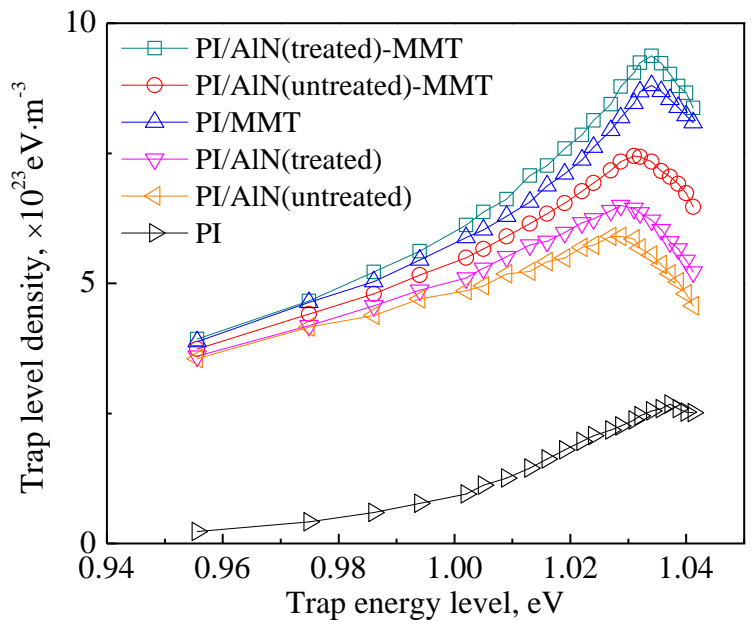

b

Fig. 4. a-IDC-time characteristics curves of trap level distributions; $b$ - five kinds of nanocomposite films

Table 2. The trap level and density of PI and nanocomposite films

\begin{tabular}{|c|c|c|c|c|c|c|}
\hline Films & PI & $\begin{array}{c}\mathrm{PI} / \mathrm{MM} \\
\mathrm{T}\end{array}$ & $\begin{array}{c}\mathrm{PI} / \mathrm{Al} \\
\mathrm{N} \\
(\mathrm{tr})\end{array}$ & $\begin{array}{c}\mathrm{PI} / \mathrm{Al} \\
\mathrm{N} \\
(\mathrm{untr})\end{array}$ & $\begin{array}{c}\mathrm{PI} / \mathrm{AlN} \\
(\mathrm{tr}) \\
\text {-MMT }\end{array}$ & $\begin{array}{c}\text { PI/AlN } \\
\text { (untr) } \\
\text {-MMT }\end{array}$ \\
\hline$E_{\mathrm{t}}(\mathrm{eV})$ & 1.038 & 1.034 & 1.029 & 1.029 & 1.034 & 1.031 \\
\hline $\begin{array}{c}N\left(E_{\mathrm{t}}\right)\left(\times 10^{23} \mathrm{e}\right. \\
\left.\mathrm{Vm}^{-3}\right)\end{array}$ & 2.859 & 8.800 & 6.490 & 5.900 & 9.370 & 7.450 \\
\hline
\end{tabular}

The maximum TLD of the PI/AIN (untreated)-MMT films doped by $5 \mathrm{wt} \%$ is $7.450 \times 10^{23} \mathrm{eV} \mathrm{m}^{-3}$, which is lower than that of the PI/MMT but higher than those of treated and untreated PI/AIN. In addition, the maximum $\mathrm{TLD}=9.370 \times 10^{23} \mathrm{eV} \mathrm{m}^{-3}$ of the PI/AIN (treated)-MMT 
film is higher than those of any other nanocomposite films, indicating that the compatibility and synergistic effect of PI and layered MMT or treated AlN nanoparticles are enhanced, i.e., the surface state of the treated AIN nanoparticles significantly increases the TLD.

\subsection{Effect of surface state on TLD}

The IDC experimental results show that variation of the surface state of AlN nanoparticles induced by the coupling agent results in a significant TLD increase of the PI/AIN (treated) film, as compared to that of the untreated one. The interface interaction zones between nanoparticles with larger surface area and polymer are formed due to stronger interface interaction between them caused by treated nanoparticles [9], so that the cluster formation is inhibited and the dispersions in PI matrix are enhanced. Based on the charge decay theory, the distribution of space charge injected into the nanocomposite film is changed, and the interface trap effects are enhanced. The TLD of PI/AIN (treated)-MMT film is much higher than those of the PI/AlN (untreated)-MMT and PI/AIN (treated) films. According to the analysis of space configuration defects for polymer chains and of the observed synergistic effect between MMT and treated AlN nanoparticles, traps are generated in the interface zone of polymer matrix inorganic nanocomposite dielectrics [15].

\section{CONCLUSIONS}

Based on the in-situ polymerization process, a pure PI film and five types of nanocomposite films, including PI/AIN (treated), PI/AIN (untreated), PI/MMT, PI/AIN (treated)-MMT, PI/AIN (untreated)-MMT, were prepared in this study. The surface state effect of inorganic nanoparticles on traps of nanocomposite films was explored by the AFM, SEM, TEM, and IDC methods. Within framework of the charge decay theory, a relationship between IDC and TLD of the nanocomposite films was determined, and the equation interrelating trap level and density was derived. The SEM and TEM results show that the surface state of AlN nanoparticles treated by the coupling agent promotes their combination with the polyimide matrix and improves the compatibility and dispersion, as compared with the untreated AlN nanoparticles. The maximum TLD of the PI/AIN (treated) is $6.490 \times 10^{23} \mathrm{eV} \mathrm{m}^{-3}$, which is higher than that of untreated one and is about 2.27 times higher than that of pure PI film in the range of $0.9 \sim 1.1 \mathrm{eV}$. The maximum TLD of PI/AIN (untreated)-MMT film is lower than that of the PI/MMT, but higher than those of treated and untreated PI/AIN films. However, the TLD of PI/AIN (treated)-MMT film doped by $5 \mathrm{wt} . \%$ is $9.370 \times 10^{23} \mathrm{eV} \mathrm{m}^{-3}$, which is higher than those of any other nanocomposite films, indicating that the interface trap effect of doped nanoparticles in PI matrix is enhanced by the surface state of treated nanoparticles, and is also influenced by the synergistic effect between the MMT and treated AlN nanoparticles.

\section{Acknowledgments}

This study was supported by the Natural Science Foundation of China (Grant Nos. 51777047 and 51337002), the China Postdoctoral Science Foundation (General Financial Grant No. 2018M630339), Key Laboratory of Engineering Dielectrics and Its Application (Harbin University of Science and Technology) and Ministry of Education (Grant No. KF20171111), and this work was also by the BSRF, Beijing, China (Grant No. 2016-BEPC-PT-001134).

\section{REFERENCES}

1. Modesti, M., Lorenzetti, A., Bon D., Besco, S. Effect of Processing Conditions on Morphology and Mechanical Properties of Compatibilized Polypropylene Nanocomposites Polymer 46 (23)

2005: pp. $10237-10245$.

https://doi.org/10.1016/j.polymer.2005.08.035

2. Khun, N.W., Loong, P.Y., Liu, E.J., Li, L. Enhancing Electrical and Tribological Properties of Poly(methyl methacrylate) Matrix Nanocomposite Films by Co-incorporation of Multiwalled Carbon Nanotubes and Silicon Dioxide Microparticles Journal of Polymer Engineering $36(1) \quad$ 2016: pp. 23-30. https://doi.org/10.1515/polyeng-2014-0346

3. Liu, X.X., Yin, J.H., Kou, Y.J., Chen, M.H., Liu, Y.Y., Li, J.L., Zhang, N., Yao, L., Wu, Z.H., Su, B. Mechanical Property of Polyimide Nanocomposite Films with Nitrogen Doped Graphene Nanoscience and Nanotechnology Letters 7 (10) 2015: pp. 262-267. https://doi.org/10.1166/nnl.2015.1957

4. Chao, D., Liang, P., Chen, Z., Bai, L.Y., Shen, H., Liu, X.X., Xia, X.H., Zhao, Y.L., Savilov, S.V., Lin, J.Y., Shen, Z.X. Pseudocapacitive Na-ion Storage Boosts High Rate and Areal Capacity of Self-branched 2D Layered Metal Chalcogenide Nanoarrays ACS Nano $10 \quad$ 2016: pp. 10211-10219.

https://doi.org/10.1021/acsnano.6b05566

5. Sun, D., Yin, J.H., Liu, Y.Y., Liu, X.X. The Electrical and Thermal Properties of Polyimide/boron Nitride Nanocomposite Films Journal of Polymer Research 23 2016: pp. 254-258. https://doi.org/10.1007/s10965-016-1151-x

6. Liu, X.X., Chao, D.L., Su, D.P., Liu, S.K., Chen, L., Chi, C.X., Lin, J.Y., Shen, Z.X., Zhao, J. P., Mai, L.Q., Li, Y. Graphene Nanowires Anchored to 3D Graphene Foam via Self-assembly for High Performance $\mathrm{Li}$ and $\mathrm{Na}$ Ion Storage Nano Energy 37 2017: pp. 108-117. https://doi.org/10.1016/j.nanoen.2017.04.051

7. Tanaka, T., Kozako, M., Fuse, N., Ohki, Y. Proposal of a Multi-core Model for Polymer Nanocomposite Dielectrics IEEE Transactions on Dielectrics and Electrical Insulation 12 (4) 2005: pp. 669-681.

8. Du, B.X., Li, J., Du, W. Surface Charge Accumulation and Decay on Direct-fluorinated Polyimide $/ \mathrm{Al}_{2} \mathrm{O}_{3}$ Nanocomposites IEEE Transactions on Dielectrics and Electrical Insulation 20 (5) 2012: pp. 1764-1771.

9. Roy, M., Nelson, J.K., MacCrone, R.K, Schadler, L.S., Reed, C.W., Keefe, R. Polymer Nanocomposite Dielectrics-the Role of the Interface IEEE Transactions on Dielectrics and Electrical Insulation 12 (4) 2005: pp. $629--641$. 
https://doi.org/10.1109/TDEI.2005.1511089

10. Simmons, J.G., Tarn, M.C. Theory of Isothermal Currents and the Direct Determination of Trap Parameters in Semiconductors and Insulators Containing Arbitrary Trap Distributions Physical Review B 7 (8)

1973: pp. 3706-3713.

https://doi.org/10.1103/PhysRevB.7.3706

11. Tian, F.Q., Bu, W.B., Yang, C., He, L.J., Wang, Y., Wang, X., Lei, Q.Q. A New Method for Direct Determination of Trap Level Distribution from TSC Measurement IEEE 9th International Conference on the Properties and Applications of Dielectric Materials, Harbin, China, 2009: pp. 980-983. https://doi.org/10.1109/ICPADM.2009.5252276

12. Lin, J.Q., Xie, Z.B., Yang, W.L., Zhang, P.P., Liu, Y., Lin, H., Li, X.K. Effect of $\mathrm{K}_{0.5} \mathrm{Na}_{0.5} \mathrm{NbO}_{3}$ Filling Particles on the Charge Carrier Trap Distribution of Polyimide Films
Journal of Applied Polymer Science

$131(3)$

2014: pp. $39828(1-7)$.

https://doi.org/10.1002/app.39828

13. Watson, P.K. The Transport and Trapping of Electrons in Polymers IEEE Transactions on Dielectrics and Electrical Insulation 2 (5) 1995: pp. 21-27. https://doi.org/10.1109/94.469986

14. Tian, F.Q., Bu, W. B., Shi, L.S., Yang, C., Wang, Y., Lei, Q.Q. Theory of Modified Thermally Stimulated Current and Direct Determination of Trap Level Distribution Journal of Electrostatics 69 (1)

2011: pp. $7-10$. https://doi.org/10.1016/j.elstat.2010.10.001

15. Feng, Y., Yin, J.G., Chen, M.H., Liu, X.X., Su, B., Fei, W.D., Lei, Q.Q. Influence of Interface on the Electrical Properties of Polyimide/ $\mathrm{TiO}_{2}$ Composite Films IEEE Transactions on Dielectrics and Electrical Insulation 21 (4) 2014: pp. 1501-1506.. 